\title{
Threonine Phosphorylation Site
}

National Cancer Institute

\section{Source}

National Cancer Institute. Threonine Phosphorylation Site. NCI Thesaurus. Code C14058.

It refers to amino acid threonine residue that was covalently modified by phosphate group (PO4-). ( $\mathrm{NCl})$ 